\title{
Factors associated with Distal Radio-UInar Joint (DRUJ) instability in patients with Distal Radius Fractures
}

\author{
Niraj Ranjeet ${ }^{1}$, Krishna Sapkota', Pratyenta Raj Onta ${ }^{2}$, Pabin Thapa ${ }^{2}$, \\ Krishna Wahegoankar ${ }^{3}$, Upendra Jung Thapa ${ }^{2}$ \\ ${ }^{1}$ Assistant Professor, Department of Orthopedics, Manipal Teaching Hospital (MTH), Pokhara, Nepal, ${ }^{2}$ Lecturer, \\ Department of Orthopedics, MTH, Pokhara, Nepal, ${ }^{3}$ Associate Professor, Department of Orthopedics, MTH, Pokhara, \\ Nepal
}

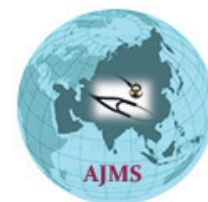

A B S T R A C T

Background: Distal Radius Fractures (DRF) are common fractures with variety of complications if treated inadequately. Among them, Distal Radio-Ulnar Joint (DRUJ) instability is one of the common residual disabilities leading to chronic and persistent subluxation or dislocation of the DRUJ which causes arthritis, pain and limitation of the hand functions. Aims and Objectives: This study aims to identify the factors either clinical of radiographic, indicative of DRUJ instability patients in acute DRF which may help the orthopedic surgeons to correctly diagnose and allow proper treatment. Materials and Methods: All patients from Aug 1, 2016-July 31, 2017 with acute DRF who were treated at Manipal Teaching Hospital were examined and evaluated for DRUJ instability. The demographic, clinical and radiological parameters of all the patients were analyzed and compared with those with stable DRUJ. Preoperatively all the fractures were classified using AO classification. Based on the classification, the association of fracture comminution, and intra-articular involvement with DRUJ instability, as well as its relation to the osteoporotic age was analyzed. Associated ulna styloid fracture and sigmoid notch involvement if present was noted with its size and displacement. Results: Out of 74 wrists, there were 30 males and 44 females, with a mean age of 44 (range 18-79). Most of the females $(72 \%, n=32 / 44)$ sustained low energy trauma, while most of the males $(77 \%, n=23 / 30)$ sustained high energy trauma. Irrespective of the sex, it was noted that DRUJ instability was significantly higher in males $(p<0.0001)$ and in the non-osteoporotic age group $(p=0.05)$. In our study $72.72 \%$ of DRF was associated with unstable DRUJ in patients with high energy trauma. AO types A3/B3/C3 $181.25 \%$, $n=26 / 32$ ) was associated with more DRUJ instability compared to less comminuted fractures as $A O$ type $A 2 / B 2 / C 2(56 \%, n=14 / 25)$ or $A 1 / B 1 / C 1(29.41 \%, n=5 / 17)(p<0.001)$. AO type $C(43.24 \%, n=32)$ were associated with more DRUJ instability as compared to $A O$ type $B(25.67 \%, n=19)$ or AO type $A(31.08 \%, n=23)(p=0.009)$. Ulnar styloid fractures was noted in $37.83 \%(28 / 74)$ of DRF and $35(47.29 \%)$ patients had involvement of the sigmoid notch out of which $7(20 \%)$ was displaced $>2 \mathrm{~mm}$ and $28(80 \%)$ was undisplaced. Conclusion: Factors associated with DRUJ instability in DRF were young male patients, high energy trauma, displaced ulnar styloid base fractures, sigmoid notch involvement, AO type C.

Access this article online

Website:

http://nepjol.info/index.php/AJMS DOI: 10.3126/ajms.v9i5.20468 E-ISSN: 2091-0576

P-ISSN: $2467-9100$

Key words: Distal radius fractures; DRUJ; Instability

\section{INTRODUCTION}

Distal Radius Fractures (DRF) is one of the most common fractures of the upper extremity with reported overall incidence from 280-440 per 100000 populations annually. Among the various complications of DRF, Distal RadioUlnar Joint (DRUJ) instability is one of the common residual disabilities with an incidence of $8-37 .^{1-3}$ Untreated 
DRUJ instability may lead to chronic and persistent subluxation or dislocation of the DRUJ and may complicate to arthritis, pain and limitation of the hand and forearm range of motion. ${ }^{1,4}$

With a history of significant trauma, high index of suspicion, thorough clinical examination and radiographs are required to diagnose DRUJ instability. But if there is an associated ulnar styloid fracture along with DRF, than there is a high chance of associated DRUJ instability. ${ }^{5,6}$ Unfortunately, assessment of DRUJ instability is incompletely discussed in most of the orthopedic textbooks. ${ }^{7}$ The lack of awareness and information of DRUJ instability in patients with acute DRF may be the reason for the documentation of low incidence rates of DRUJ instability in the literature.

This study aims to identify the factors either clinical or radiographic, indicative of DRUJ instability in patients with acute DRF which may help the orthopedic surgeons to correctly diagnose and allow proper treatment.

\section{MATERIALS AND METHODS}

From Aug 1, 2016-July 31, 2017, all patients with acute DRF who attended Manipal Teaching Hospital were examined and evaluated.

Inclusion criteria included patients $>18$ years old with $\mathrm{DRF}<3$ weeks old. Exclusion criteria included patients $<18$ years old and DRF $>3$ weeks old and fracture configuration that could complicate interpretation of instability and associated carpal bone fractures. Ethical clearance was obtained from the ethical committee of our hospital before commencement of this study.

All patients with DRF were reduced either closed or open followed by reduction maintenance in either plaster, pins and plaster, external fixator or Open reduction and internal fixation (ORIF). Following the procedure a piano key test and range of motion of the wrist were done intraoperatively and a standard x-ray of wrist PA and lateral were done to assess the DRUJ instability. DRUJ instability was diagnosed if there was a positive piano key test (gross dislocation of DRUJ on pronation or supination) or there is a gross dislocation of the DRUJ on standard wrist x-ray. The piano key test is performed first on the normal wrist then followed by the injured wrist. The test is performed with the wrist in neutral, pronation and supination. The elbow in placed on the table at 90 degrees. The examiner stabilizes the distal radius with one hand and the other hand is used to translate the distal ulna both dorsally and volarly. The test is called positive if there is increased dorsal or volar translation of the ulna as compared to the contralateral wrist. Instability is not considered as the criteria if there is a subluxation of the DRUJ on wrist $\mathrm{x}$-ray without any supporting clinical findings.

The demographic, clinical and radiological parameters of all the patients with DRUJ instability in acute DRF was analyzed and compared with those with stable DRUJ. The incidence of DRUJ instability in patients with DRF was established. Preoperatively all the patient with acute DRF was analyzed and classified using AO classification. Based on the classification, the association of fracture comminution and intra-articular involvement with DRUJ instability was analyzed, as well as its relation to the osteoporotic age (males $>60$, females $>50$ ).

After the standard radiograph the x-ray parameters (radial height, radial tilt, volar/dorsal tilt, ulnar variance, step and gap) were measured for each fracture and they were classified as either displaced or acceptable. Associated ulna styloid fracture if present was noted with its size and displacement. Associated sigmoid notch involvement if present was noted with its displacement. Associated fracture displacement if present was correlated with the presence of DRUJ instability.

All the available literature was reviewed for prevalence of risk factors for DRUJ instability in acute DRF. The sample size of the study needed to detect the relationship of osteoporotic fractures with DRUJ instability (the smallest difference among risk factors at $32 \%$ ) at alpha 0.05 and power of $80 \%$ was computed to be 50 .

\section{RESULTS}

From the period of August 1, 2016-July 31, 2017, 83 patients with a total of 93 acute DRF were included in our study. Five patients had involvement in both the wrist. Nine patients were excluded from our study because of incomplete demographic data or radiographs bringing the total to 74 wrists, including 3 patients with bilateral wrist involvement.

Male to female ratio was 0.68:1 (M:30, F:44), with a mean age of 44 (range 18-79). Majority (76\%) of the females were at osteoporotic age (mean 52, range 18-79), while only 5 males had osteoporosis (mean 35, range 18-64).

Mechanisms of injury, as classified into low energy (fall at standing height) or high energy (fall from height, motorvehicular accident) were noted. As described in literatures, most of the females $(72 \%, \mathrm{n}=32 / 44)$ sustained low energy trauma, while most of the males $(77 \%, \mathrm{n}=23 / 30)$ sustained high energy trauma. 
The incidence of DRUJ instability was $22.9 \%(\mathrm{n}=17 / 74)$, and majority $(83.33 \%, n=15 / 18)$ of the patients were males. There were three open DRF that were associated with DRUJ instability in our study.

Irrespective of the sex, it was noted that DRUJ instability was significantly higher in males $(p<0.0001)$ and in the non-osteoporotic age group $(p=0.05)$. But when the ceiling for the osteoporotic age was reduced to 50 for either sexes, a similar trend toward occurrence of DRUJ instability was shown though it was insignificant $(\mathrm{p}=0.09)$.

In our study $72.72 \%$ of DRF was associated with unstable DRUJ in patients with high energy trauma. It was noted that DRUJ instability was more common in patients with high energy fractures (motorbike accidents, fall from height) $(72.72 \%, \mathrm{n}=16 / 22)$ as compared to low energy fractures (fall at standing height) $(36.5 \%, \mathrm{n}=19 / 52)$ $(\mathrm{p}<0.001)$.

The amount of comminution as classified according to AO classification had an association with DRUJ instability. Highly comminuted fractures as classified to AO types A3/ B3/C3 (81.25\%, $\mathrm{n}=26 / 32)$ was associated with more DRUJ instability compared to less comminuted fractures as $\mathrm{AO}$ type A2/B2/C2 (56\%, $\mathrm{n}=14 / 25)$ or A1/B1/C1 $(29.41 \%$, $\mathrm{n}=5 / 17)(\mathrm{p}<0.001)$ (Figure 1). It was also seen that intraarticular fractures, as classified to AO type C (43.24\%, $\mathrm{n}=32)$ were associated with more DRUJ instability as compared to AO type B $(25.67 \%, \mathrm{n}=19)$ or AO type A $(31.08 \%, \mathrm{n}=23)(\mathrm{p}=0.009)$.

An ulnar styloid fracture was noted in $37.83 \%$ (28/74) of DRF. Out of them 18 had base of ulna styloid and 10 had tip of ulna styloid fractures (Figure 2). The association of ulna styloid fracture by itself was not associated with DRUJ instability $(\mathrm{p}=0.4)$, but the presence of ulna styloid base fracture as compared to body fracture was significantly associated with DRUJ instability $(\mathrm{p}=0.04)$. Out of these styloid fractures, 19 were displaced $>2 \mathrm{~mm}$ while 9 were undisplaced. Displaced fracture more than $2 \mathrm{~mm}$ were significantly associated with DRUJ instability as compared to un-displaced ulna styloid fracture $(\mathrm{p}=0.002)$.

Of the total DRF, $35(47.29 \%)$ patients had involvement of the sigmoid notch out of which $7(20 \%)$ was displaced $>2 \mathrm{~mm}$ and $28(80 \%)$ was undisplaced (Figure 3). 6 out of $7(86 \%)$ patients with displaced sigmoid notch was associated with DRUJ instability as compared to 13 out of $28(46 \%)$ patients with un-displaced sigmoid notch $(\mathrm{p}=0.001)$.

After an univariate analysis, factors that were found to be significantly associated with DRUJ instability are

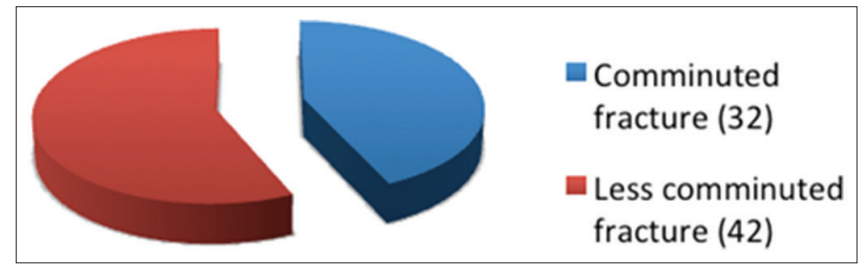

Figure 1: Variables according to comminution

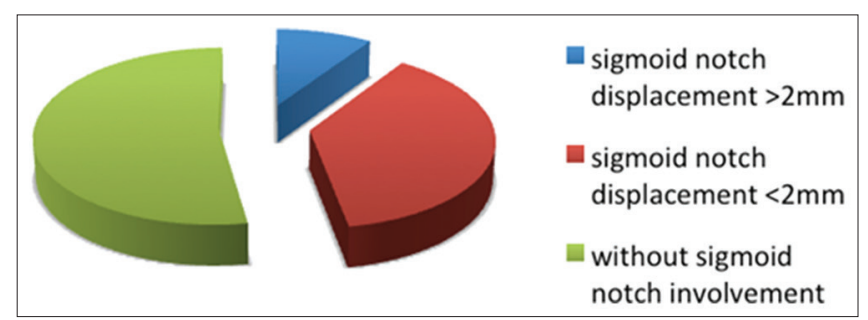

Figure 2: Variables according to ulna styloid fracture

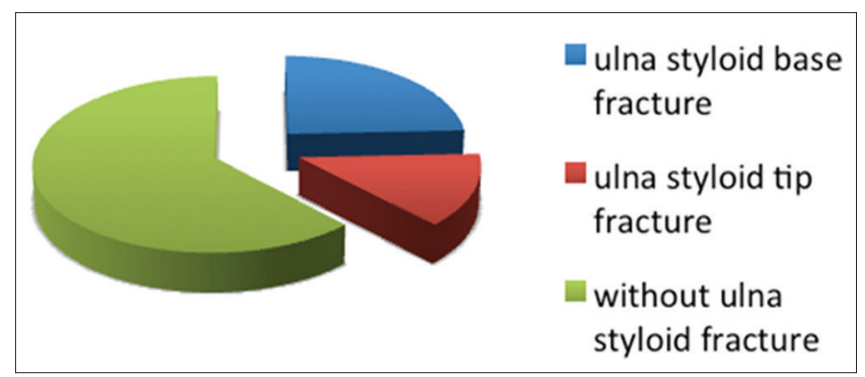

Figure 3: Variables according to involvement of sigmoid notch

\begin{tabular}{lc}
\multicolumn{2}{l}{ Tables 1: Demographic data of population } \\
\hline Variables & Percentage \\
\hline Sex & $30(40.54)$ \\
Male & $44(59.46)$ \\
Female & \\
Age & $8(26.66)$ \\
Male $>59$ years & $22(73.34)$ \\
Male $<59$ years & $15(34.1)$ \\
Female $>49$ years & $29(65.9)$ \\
Female $<49$ years & \\
Mechanism of Injury & $52(70.27)$ \\
Fall at standing height & $9(12.16)$ \\
Fall from height & $13(17.57)$ \\
Road traffic accidents & \\
AO Classification & $32(43.24)$ \\
A & $19(25.67)$ \\
B & $23(31.09)$ \\
C
\end{tabular}

mentioned in table 1 . These factors were further analyzed using multivariate analysis. On multiple regression, the only significant association for occurrence of DRUJ instability were male gender and non-osteoporotic age. To analyze the effect of displacement of these fractures, a separate logistic regression model was used with the data from patients with ulnar styloid base fractures. The analysis showed that ulnar styloid base fracture with displacement $>2 \mathrm{~mm}$ was significantly associated with DRUJ instability (OR 6.77e+08). 


\section{DISCUSSION}

DRUJ instability negatively affects the outcomes in patients with DRF as it leads to loss of hand and wrist function. ${ }^{2,8,9}$ Patients with DRF with DRUJ instability had significantly weak hand grips, significant pain and tenderness and poor range of motions of hand and wrist.

Despite the correlation between DRF and DRUJ instability, orthopedic surgeons still tend to overlook the importance of DRUJ instability and its management in cases of acute DRF. As compared to its importance very little research publication has been done and it feels that the clinical entity has been neglected in the acute DRF.

DRUJ injury usually occurs after a hyperpronation injury leading to dorsal dislocation or a hypersupination injury leading to volar dislocation. ${ }^{10}$ These injuries are sustained after a fall on an outstretched hand either from a standing height or road traffic accidents. ${ }^{11}$

DRUJ instability is more associated with high energy trauma secondary to road traffic accidents. Depending on the severity of injury, DRUJ injury may vary from a simple avulsion fracture of the tip of the ulna styloid to comminuted fracture at the base of the ulna styloid. ${ }^{1}$

The base of ulna styloid provides the important attachment for triangular fibrocartilage complex (TFCC) so its fracture leads to DRUJ instability. The TFCC does not attach uniformly to ulnar styloid. The volar and dorsal radio-ulnar ligaments are the major stabilizers for DRUJ and are attached to the base and to the fovea of the ulnar styloid. The size and site of the fracture fragment and the amount of displacement are both risk factors for DRUJ instability. ${ }^{5}$ The larger the fragment and the nearer the fracture to the base of the styloid, the more likely it may include the attachment of the TFCC. In a retrospective study, analyzing DRUJ instability in patients with DRF, fractures involving the base of the ulnar styloid and those fractures with displacement $>2 \mathrm{~mm}$ were found to have increased risk of DRUJ instability. ${ }^{3}$ As observed in our study, ulnar styloid fractures as such does not suggest DRUJ instability but if there is ulnar styloid base fractures or ulnar styloid fractures displaced $>2 \mathrm{~mm}$ DRUJ instability were predictable.

We found no previous clinical studies that have proven the association of sigmoid notch involvement in the association of DRUJ instability in patients with DRF. Morrisey et al in 2006 performed a biomechanical cadaveric study and observed DRUJ instability on various degrees of TFCC tears in association with dorsal and volar lunate facet fractures. ${ }^{10} \mathrm{In}$ our study, we observed that if the sigmoid notch which gives the attachment to the volar and dorsal radio-ulnar ligaments, was involved it leads to DRUJ instability, especially when displaced >2mm. In 1996 Geissler et al conducted a study where arthroscopic findings in patients with intra-articular DRF were noted and found that most $\mathrm{AO}$ type $\mathrm{C}$ and those involving the lunate facet were associated with TFCC tears and other intra-carpal soft tissue injuries. ${ }^{1}$

In patients with normal bone stock, trauma to the wrist causes ligamentous injury of the TFCC as compared to osteoporotic patients, where avulsion fractures of the ulnar styloid is more common. In 2000, Lindau et al. found a higher incidence (37\%) of DRUJ instability in patients with DRF with normal bone (males $<60$, females $<50$ years old) as compared to other studies which included mostly osteoporotic fractures. ${ }^{1,3}$ These study suggests that DRUJ instability includes a spectrum of osseous and soft tissue injury.

There are some radiological parameters, which are suggestive of DRUJ instability in literature. They include widening of the DRUJ space $>2 \mathrm{~mm}$ compared to the contralateral wrist, positive ulnar variance $>2 \mathrm{~mm},>5 \mathrm{~mm}$ of proximal migration of the distal radius and $>20^{\circ}$ of dorsal angulation. ${ }^{6,11-13}$ In our study we were able to find some trends with regards to the radiological parameters but we were not able to prove it to be significantly associated with the occurrence of DRUJ instability.

In the univariate analysis several variables were found to be significantly associated with DRUJ instability, but when multivariate analysis was done only male gender and patients with non-osteoporotic age, showed significant association with DRUJ instability. Our results showed that DRF with both sigmoid notch involvement and ulnar styloid base fractures was not associated with DRUJ instability. This could be explained by interaction between the variables involved. Individual factors alone may be associated with an increased risk for DRUJ instability but in vivo they may not be compatible with the development of instability as suggested by our data. Given that instability can occur in a spectrum of bony and ligamentous injury, certain fracture configurations may not predispose to DRUJ instability. A non-osteoporotic fracture with a concomitant ulnar styloid base fracture would indicate an associated tear of the attachment of the TFCC, the primary stabilizing component of the DRUJ, leading to instability. If the sigmoid notch were also involved, this could point to a purely osseous injury, with most of the traumatic energy being dessipated through the bone, thus sparing the TFCC.

Though we were able to meet the sample size, there was a predominance of certain variables (males with high energy trauma, females with osteoporotic fractures) in our study, which might possibly have affected the statistical analysis. Also, due to lack of enough data in literature not all of the variables were factored in computing the sample size 
in our study. Thus, the computed sample size might have been underestimated, with not enough power to detect a possible smaller difference among the variables.

In our study, we only evaluated the DRUJ instability using clinical and radiographic means. Criteria for evaluating the DRUJ injury using CT scans have been established. CT scan has been advocated as a non-invasive study to accurately demonstrate subluxation and dislocation of the DRUJ. ${ }^{6}$

\section{CONCLUSION}

In patients with DRF, DRUJ injury is a commonly associated injury and must be always considered and evaluated. Males at non-osteoporotic age with DRF secondary to high energy trauma are likely to be associated with DRUJ instability. Patients with intra-articular fractures, comminuted fractures, presence of ulnar styloid base fractures, involvement of sigmoid notch, were associated risk factors for DRUJ instability in acute DRF. These identified factors will guide us to recognize DRUJ instability in patients with DRF and allow its early management.

\section{REFERENCES}

1. Geissler W, Freeland A, Savoie F, Mclntyre L and Whipple T. Intracarpal Soft Tissue Lesions Associated with Intra-articular Fracture of the Distal Radius. The Journal of Bone and Joint Surgery 1996; 78A:357-365.

2. Lindau T, Adlercreutz $C$ and Aspenburg P. Peripheral tears of the triangular fibrocartilage complex cause distal radioulnar joint instability after distal radius fractures. The Journal of Hand Surgery 2000; 25A(3):464-468.

3. May M, Lawton J and Blazar P. Ulnar styloid fractures associated with distal radius fractures: incidence and implications for distal radioulnar joint instability. The Journal of Hand Surgery 2002; 27A(6):965-971.

4. Bruckner $\mathrm{J}$, Alexander $\mathrm{H}$ and Lichtman D. Instructional Course Lectures, The American Academy of Orthopedic Surgeons. Acute dislocations of the distal radio-ulnar joint. The Journal of Bone and Joint Surgery 1996. 77A (6):448-458.

5. Hauck R. Ulnar styloid fractures: A review. Current Opinion in Orthopedics 2005; 16:227-230.

6. Szabo R. Distal Radioulnar Joint Instability. The Journal of Bone and Joint Surgery 2006; 88A: 884-894.

7. BrucknerJ, Lichtman $\mathrm{D}$ and Alexander H. Complex dislocations of the distal radioulnar joint. Clinical Orthopedics and Related Research 1992; 275:90-103.

8. Lindau T, Hagberg L, Adlercreutz C, Jonsson K and Aspenburg P. Distal radioulnar instability is an independent worsening factor in distal radius fractures. Clinical Orthopedics and Related Research 2000; 376:229-235.

9. Stoffelen $D$, De Smet $L$ and Broos P. The importance of distal radioulnar joint in distal radius fractures. The Journal of Bone and Joint Surgery 1998. 23B:507-511.

10. Morrisey $R$ and Nalebuff $M$. Dislocation of the distal radioulnar joint: Anatomy and clues to diagnosis. Clinical Orthopedics and Related Research 1979. 144:154-158.

11. Shin A, Deitch M, Sachar K and Boyer M. Ulnar sided wrist pain. Diagnosis and treatment. The Journal of Bone and Joint Surgery 2004; 86A:1560-1574.

12. Lindau $T$ and Aspenburg $P$. The radioulnar joint in distal radius fractures. Acta Orthopedica Scandinavia 2002; 73(5):579-588.

13. Lindau T, Runnquistz and Aspenburg P. Patients with laxity of distal radioulnar joint after distal radius fractures have impaired function, but no loss of strength. Acta Orthopedica Scandinavia 2002; 73(5):151-156.

\footnotetext{
Authors Contribution:

NR - Concept and design, review of literature, statistically analysis and interpretation, manuscript preparation and revision; KS- Literature search and review PRO - Data collection and manuscript revision; PT- Data collection, statistically analysis and literature review; KW- Manuscript revision and literature review; UJT- Literature search and review.

Work Attributed to: Department of Orthopedics, Manipal Teaching Hospital, Phulbari, Pokhara, Nepal.

Orcid ID:

Dr. Niraj Ranjeet - (1) https://orcid.org/0000-0001-5460-5839

Dr. Krishna Sapkota - io https://orcid.org/0000-0002-2277-8556

Dr. Pratyenta Raj Onta - https://orcid.org/0000-0002-6013-4014

Dr. Pabin Thapa - https://orcid.org/0000-0003-1336-5837

Dr. Krishna Wahegaonkar - (1) https://orcid.org/0000-0001-7274-7099

Dr. Upendra Jung Thapa - (1) https://orcid.org/0000-0003-1980-5610

Source of Support: Nil, Conflict of Interest: None declared.
} 